\title{
THE VARIABLE CURRENT GUN: THE PARAMETER TESTS AND THE RESULTS OF THE FIRST ELECTRON COOLING EXPERIMENTS AT LEAR
}

\author{
J. Bosser, R.M. Lapik*, R. Ley, I.N. Meshkov**, V.N. Poliakov**, \\ I.A.Seleznev*, A.V. Smirnov**, E.M. Syresin**, G. Tranquille, \\ A.M.Zapunjako*, M.A. Zavraznov*
}

\begin{abstract}
The electron cooling time (or equivalently the cooling force) is inversely proportional to the electron beam current $I_{b}$, aimed to cool hot lowenergy ions [1-3]. On the other hand, highly cooled ions tend to become unstable. This implies that once the ion beam upper density limit is attained, the electron current intensity has to be reduced to a level which prevents the ion beam instability and maintains low emittances. An adiabatic-type gun [4-6] has been constructed, which provides low-temperature electrons of large, but variable densities. The electron current density is adjusted through the voltage control, $U_{g}$, of a so-called grid electrode. Its main drawback is the storage of secondary electrons when the grid potential is larger than the anode potential, thus inducing a reduction of the nominal electron current intensity. A detailed analysis of the storage process is presented, and the way to cure this draw back is explained. Finally, experimental results are given.
\end{abstract}

* Centre of Applied Physics and Technology (CAPT), Lipetsk, Russia

** Joint institute for Nuclear Physics (JINR), Dubna, Russia

Paper to be published in Nuclear Instruments and Methods

Geneva, Switzerland

$21 / 6 / 94$ 


\title{
THE VARIABLE CURRENT GUN: THE PARAMETER TESTS AND THE RESULTS OF THE FIRST ELECTRON COOLING EXPERIMENTS AT LEAR
}

\author{
I.N. Meshkov, V.N. Poliakov, A.V. Smirnov, E.M. Syresin \\ Joint Institute for Nuclear Research (JNR), Dubna, Russia \\ R.M. Lapik, I.A. Seleznev,A.M. Zapunjako,M.A. Zavraznov \\ Centre of Applied Physics and Technology (CAPT), Lipetsk, Russia \\ J. Bosser, R. Ley, G. Tranquille \\ PS Division, CERN, CH 1211 Geneva, Switzerland
}

\begin{abstract}
The electron cooling time (or equivalently the cooling force) is inversely proportional to the electron beam current $I_{b}$, aimed to cool hot low-energy ions [1-3]. On the other hand, highly cooled ions tend to become unstable. This implies that once the ion beam upper density limit is attained, the electron current intensity has to be reduced to a level which prevents the ion beam instability and maintains low emittances. An adiabatic-type gun [4-6] has been constructed, which provides low-temperature electrons of large, but variable densities. The electron current density is adjusted through the voltage control, $U_{g}$, of a so-called grid electrode. Its main drawback is the storage of secondary electrons when the grid potential is larger than the anode potential, thus inducing a reduction of the nominal electron current intensity. A detailed analysis of the storage process is presented, and the way to cure this drawback is explained. Finally, experimental results are given.
\end{abstract}

\section{INTRODUCTION}

The generation of an electron beam with a low angular spread is a critical point in the design of electron guns for the cooling of ion beams [1-5]. For the LEAR electron cooling device a new electron gun has been elaborated [6] which generates an electron beam with a wide range of parameters (Table 1).

The gun (Fig.1) consists of three electrodes: the Pierce electrode, the grid and the anode. An adiabatic optics $[2,4,6]$ scheme was chosen with the peculiarity that the gun is designed to operate in a relatively low longitudinal guiding magnetic field $B$. It also offers new facilities for the operation of the LEAR electron cooler:

1. the possibility to switch on and off the electron beam without perturbing the circulating ion beam,

2. a much higher electron beam current for energies lower than $20 \mathrm{keV}$,

3 . beam current regulation during the electron cooling process. 


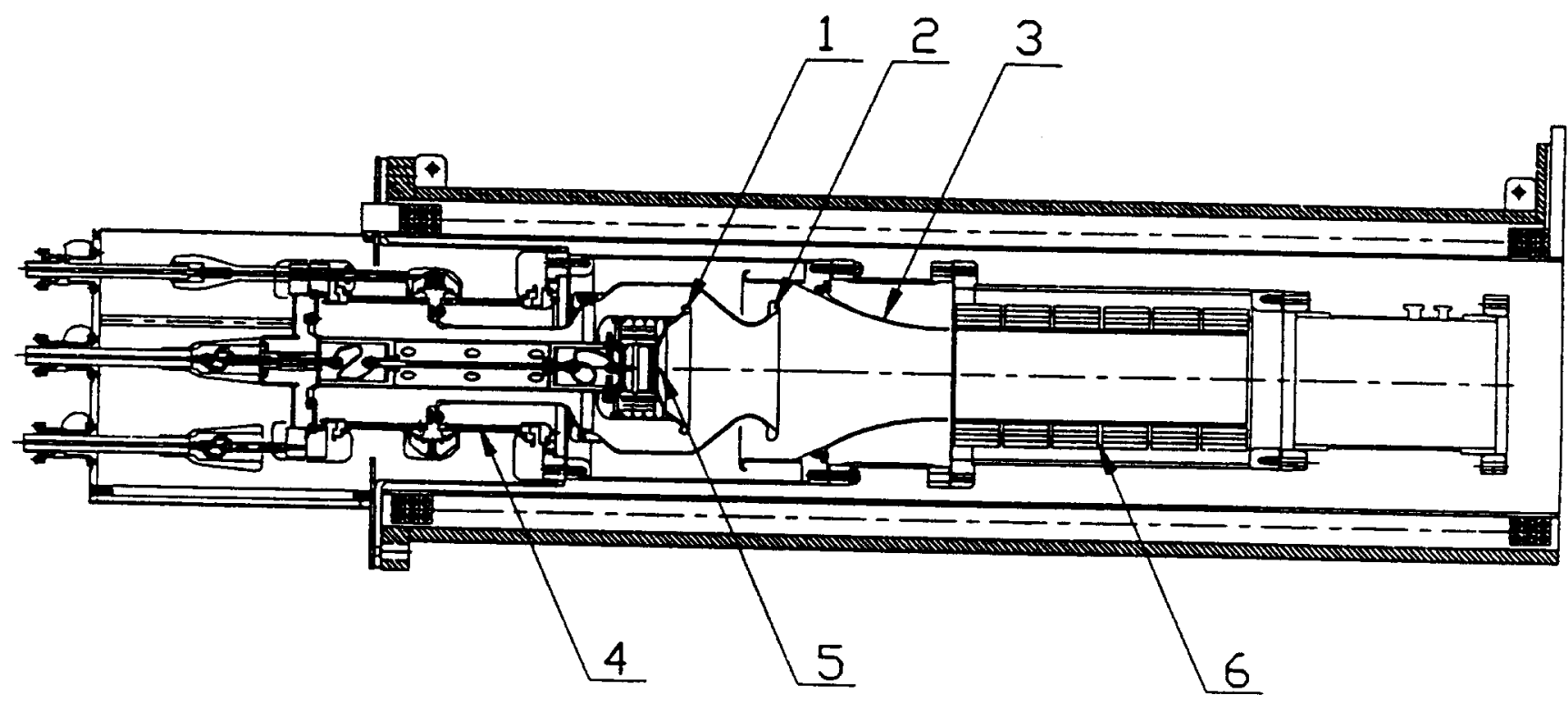

Fig. 1 - The new LEAR electron gun. 1) Pierce electrode, 2) grid, 3) anode, 4) high voltage feedthrough, 5) cathode, 6) NEG pump.

Computer simulations of the gun optics and its design were done at CAPT and at CERN at the end of 1991, and the gun was constructed during 1992 in the workshop of the Budker Institute of Nuclear Physics in Novosibirsk. The initial parameter tests were performed at CAPT between November 1992 and January 1993. The goals of the initial tests were to obtain a vacuum pressure of less than $1 \mathrm{nT}$ Torr and to generate high intensity electron beams in pulsed and stationary regimes. At the same time a measurement of the angular spread of the beam was implemented. Delivery to CERN took place at the end of January 1993, and after additional vacuum acceptance tests it was mounted on the LEAR cooler. The first electron cooling experiments with the new gun were performed in April 1993 (Table 2).

This report presents the description of the tests and a discussion of the results. The point of special interest encountered during the experiments is the regime where secondary electrons are stored in the gun when the grid potential is positive with respect to ground.

As concerns the gun polarization voltages we adopt the following conventions (Fig.2):

- The unipolar cathode power supply $U_{c}$ is always positive(or zero). This implies that the cathode is always at a negative (or zero) potential $-U_{c}$ versus ground. The electron beam velocity $v$ at the level of the grounded anode is about: $v \cong \sqrt{2 e U_{c} / m}$ where $e$ and $m$ are the electron charge and mass, respectively.

- The grid power supply $U_{g}$ is bipolar. $U_{g}$ is positive when the grid is at a positive potential versus ground (and conversely $U_{g}<0$ when the grid is at a negative potential versus ground). A current $I_{b}$ will be delivered by the gun when $U_{0}=U_{c}+U_{g} \geq 0$ or $U_{g} \geq-U_{c}$. Therefore, we will always consider the case where $U_{0}>0$ which is the sole case of physics interest. 


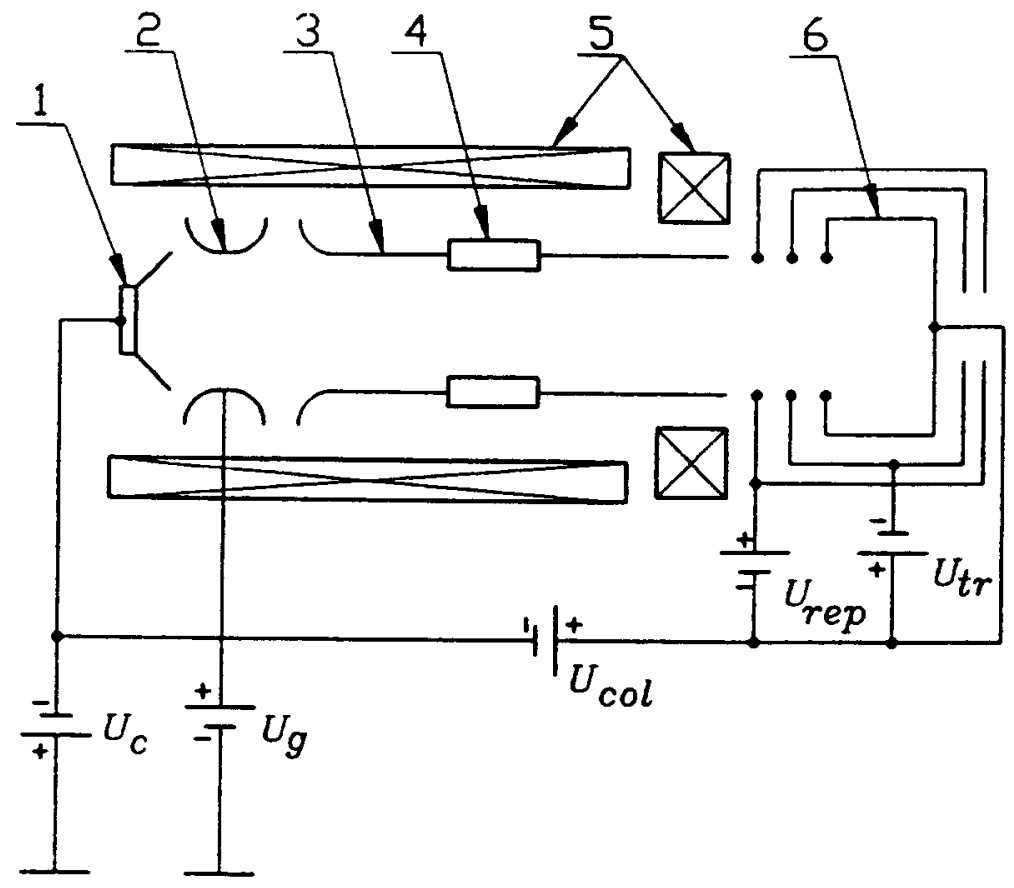

Fig. 2 - Layout of the test bench. 1) gun cathode, 2) grid, 3) anode and vacuum chamber, 4) electrostatic trap, 5) magnetic coils, 6) collector.

The new gun uses the grid for the beam current variation whilst the beam energy depends on the potential difference between the cathode and the anode and very slightly on the electron beam current through its space charge. Therefore the gun performance can be described by two parameters :

1. the gun perveance, which is given by

$$
P_{g u n}=\frac{I_{b}}{U_{0}^{3 / 2}}
$$

2. the beam perveance given by

$$
P_{\text {beam }}=\frac{I_{b}}{U_{c}^{3 / 2}}
$$

The gun perveance does not depend on the cathode voltage but only on the cathode-grid voltage difference, and the theoretical value for this gun was found to be $P_{\text {gun }}=0.56 \mu \mathrm{A} / \mathrm{V}^{3 / 2}$.

Table 1 - The electron gun parameters

\begin{tabular}{|lr|c|c|c|c|}
\hline Electron energy & $(\mathrm{keV})$ & 2.3 & 7 & 20 & 30 \\
\hline Beam current & $(\mathrm{A})$ & 0.01 to 0.53 & 0.07 to 2.93 & 0.35 to 2.83 & 0.65 to 2.6 \\
\hline Beam perveance & $\left(\mu \mathrm{A} / \mathrm{V}^{3 / 2}\right)$ & 0.125 to 5 & 0.125 to 5 & 0.125 to 1 & 0.125 to .5 \\
\hline Gun perveance & $\left(\mu \mathrm{A} / \mathrm{V}^{3 / 2}\right)$ & 0.56 & 0.56 & 0.56 & 0.56 \\
\hline Grid potential [vs. ground] $(\mathrm{kV})$ & -1.45 to 7.4 & -4.3 to 25.8 & -12.5 to 9.5 & -18.9 to -2.2 \\
\hline
\end{tabular}


Table 2. Parameters for the electron cooling tests with protons

\begin{tabular}{|c|c|}
\hline Proton beam momentum $(\mathrm{MeV} / \mathrm{c})$ & 310.1 \\
\hline Proton beam intensity & up to $2 \times 10^{10}$ particles \\
\hline Electron beam energy & 27.8 \\
\hline Electron beam current $I_{b}$ & 2.5 \\
\hline Momentum spread of cooled beam & $\pm 1.5 \times 10^{-4}$ \\
\hline Vacuum pressure & $3 \times 10^{-11}$ \\
\hline $\begin{array}{l}\text { Collector characteristics: } \\
\text { Collector potential } \\
\text { Current losses, } \Delta_{b} / I_{b}\end{array}$ & $\begin{array}{c}3.3 \\
3 \times 10^{-4}\end{array}$ \\
\hline
\end{tabular}

\section{THE TEST BENCH}

The tests at CAPT were performed on a special test bench called the "recuperator". It was constructed in 1991 for experiments with the LEAR electron beam collector [7] and has since been modernized. It consists of a vacuum chamber immersed in a $2.5 \mathrm{~m}$ long solenoid, a new electron beam collector [8] and an ion trap. The collector (Fig. 3) is a Faraday cup with a repeller electrode and an additional "transport electrode" which optimizes the beam trajectory inside the collector for intense low energy electron beams. The electric field of the repeller electrode and the beam space charge form an electrostatic barrier which prevents any secondary electrons from escaping from the collector. This effect is also amplified by the influence of a magnetic trap appearing near to the collector entrance due to the sharp decrease of the solenoid magnetic field produced by the magnetic shielding of the collector [9]. This field decrease also produces a fast expansion of the primary beam in the collector and hence reduces any space charge effects inside the collector itself.

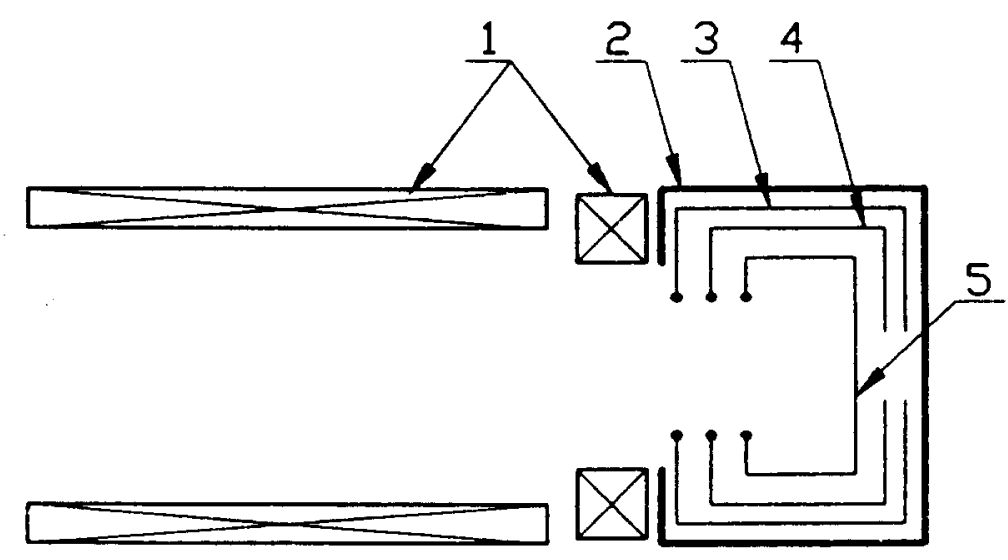

Fig. 3 - The collector schematics. 1) magnetic coils, 2) magnetic screen, 3) repeller electrode, 4) transport electrode, 5) collector. 
To diminish the influence of slow electrons stored in the gun (see section 3) an electrostatic trap [10,11] was used (part 4 in Fig.2). It consists of two halves of a metal cylinder connected together by plates of special conducting glass (Fig. 4). The positive voltage applied between the electrodes creates a transverse electric field and slow electrons arriving in the trap will drift to the glass plate and escape the system due to the crossed electric and magnetic (solenoid) field.

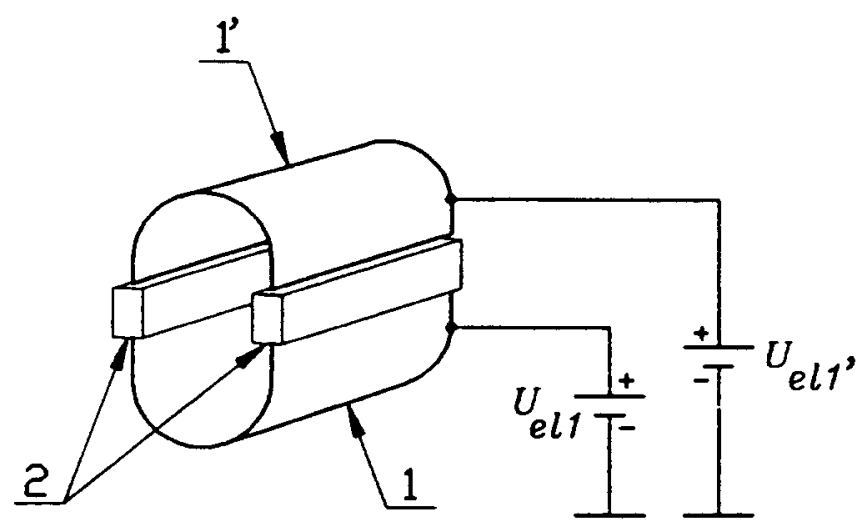

Fig. 4 - The electrostatic trap. 1,1') metallic electrode, 2) glass insulators.

The vacuum pressure in the test bench system was (after a bakeout cycle at $300^{\circ} \mathrm{C}$ ) about $1 \mathrm{nTorr}$ when the electron beam was off. The experiments with the electron beam were performed at pressures varying between $10^{-8}$ and $10^{-6}$ Torr.

The high voltage power supplies (Fig. 2) consist of one main source feeding a high voltage platform on which all the collector power supplies were installed. A separate bipolar HT supply was used for the grid which needed to be varied independently from the cathode voltage.

\section{THE PULSED MODE TESTS}

The first attempts to bring the gun into operation in a stationary regime showed that under certain conditions the beam current delivered by the gun was significantly lower than that predicted by the computer simulations. To find the reason for such a discrepancy the gun was tested in the pulsed mode regime. In this way we were able to exclude the effects of any electron storage and moreover the influence of the collector.

The gun potentials were chosen in such a way so as to stop any primary electron beam generation. In order to extract the beam (see Fig. 5) a positive voltage pulse was applied to the grid for 10 to $30 \mu$ s and the beam current was monitored. This was done for various values of the pulse amplitude and the subsequent evaluation of the gun perveance gave an exact agreement with the theoretical value of $P_{\text {gun }}=0.56 \mu \mathrm{A} / \mathrm{V}^{3 / 2}$. 


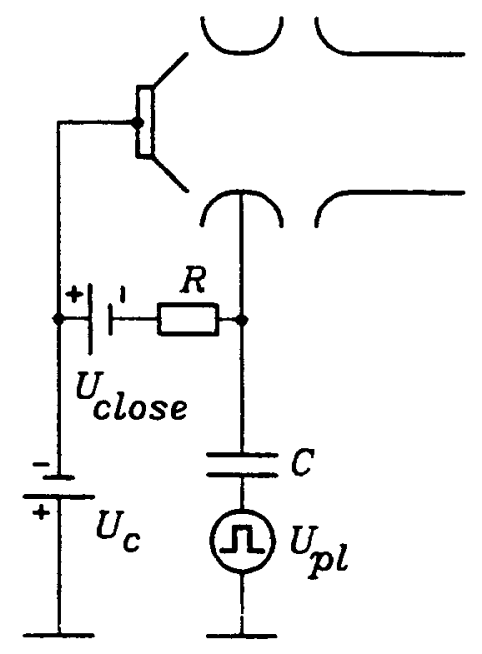

Fig. 5 - Schematics of the pulsed mode experiment. $U_{c}, U_{c l o s e}$ : cathode and grid bias power supplies, $U_{p l}$ : the pulse generator, $R C$ - a filter.

\section{THE STATIONARY REGIME TESTS}

The gun tests in the stationary regime enabled us to study the influence of the collector on the beam current, the vacuum conditions, the electrostatic trap and the focusing with the solenoid magnetic field. It was found that even for high beam perveances $\left(P_{\text {beam }}=5 \mu \mathrm{A} / \mathrm{V}^{3 / 2}\right)$ the current losses (Fig. 6) from the collector were less than $10^{-4}$, and that these losses were due to the ionization of the residual gas by the electrons [9,7]. Such a low level of current loss shows that the beam and collector perveances are in proper agreement, i.e.

$$
I_{b}=P_{\text {beam }} U_{c}^{3 / 2} \leq P_{c o l} U_{c o l}^{3 / 2}
$$

where $U_{c o l}$ is the collector potential relative to the cathode.

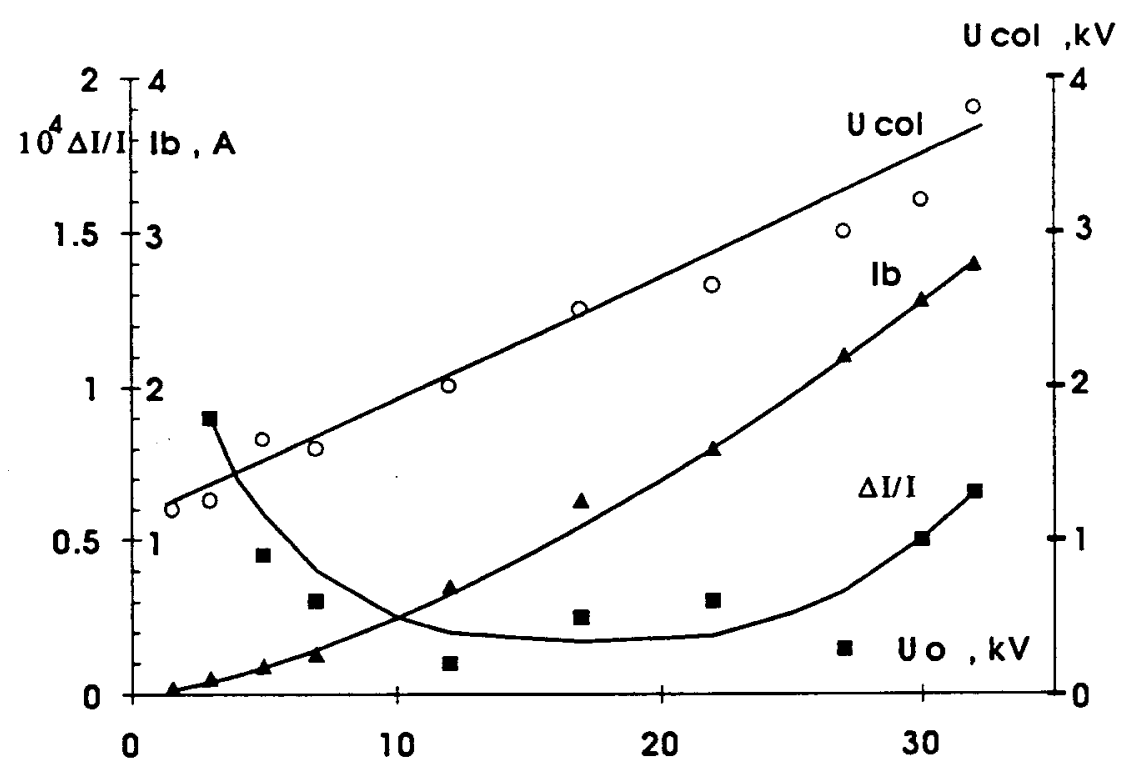

Fig. 6 - The dependence of current losses, beam current and collector potential on the extracting voltage. $P=4 \times 10^{-8} \mathrm{Torr}, U_{c}=7 \mathrm{kV}, B=480 \mathrm{G}$ and $U_{r e p}=-2$ to $-3 \mathrm{kV}$. 
During the tests two kinds of gun regimes were discovered. If the grid has a negative potential with respect to ground then the gun behaves as in the simulations and has a gun perveance of $P_{g u n}=0.56 \mu \mathrm{A} / \mathrm{V}^{3 / 2}$. However, when this potential is positive, the beam current and hence the gun perveance are much lower than the design values. From the information gathered during the pulsed mode tests, this effect was attributed to the storage of secondary electrons between the cathode and the anode. These secondary electrons are generated in collisions of the primary beam with the residual gas atoms and lead to a limitation of the beam current.

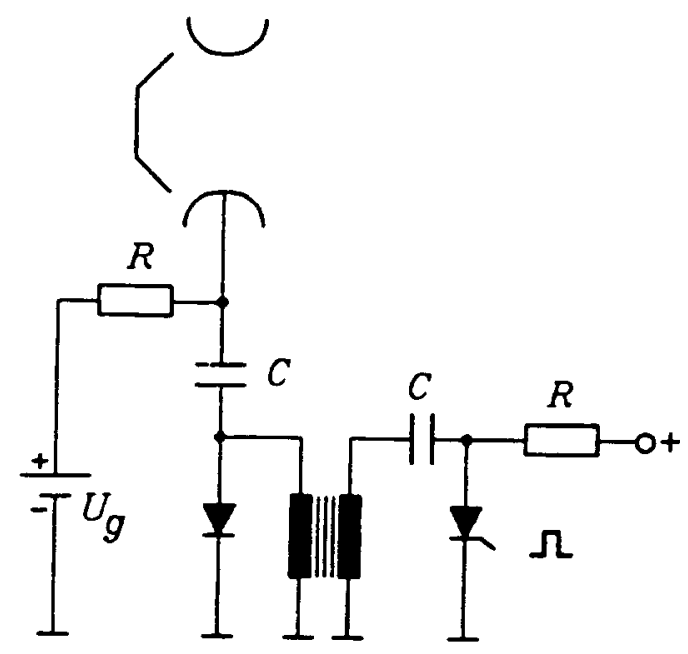

Fig. 7 - The pulse generator scheme

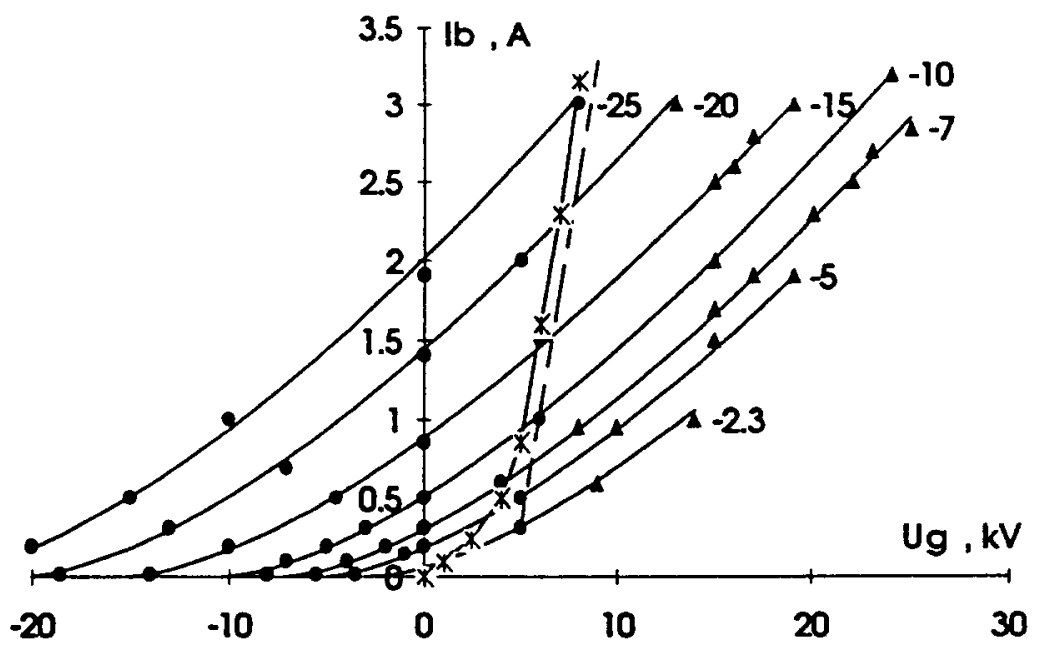

Fig. 8 - The dependence of beam current on the grid potential (with respect to ground). - experimental points when the pulser is off, $\Delta$ experimental points when the pulser is on, ---- the pulser regime boundary, $x \times x$ the theoretical boundary for the same regime.

To avoid such an undesirable effect a special pulse generator (Fig. 7) was installed. It provides periodic negative voltage pulses of $5 \mu$ s duration to the grid. This negative potential enables the stored secondary electrons to be expelled into the drift chamber and as a result the full beam current is once again obtained. The beam current dependence on the grid potential for 
different cathode voltages (Fig. 8) shows that it can be smoothly regulated from 0 to a maximum level corresponding to a beam perveance of $5 \mu \mathrm{A} / \mathrm{V}^{3 / 2}$. Figure 8 shows that there exists a definite boundary between the two regimes (dotted line) and that this threshold only depends on the cathode voltage. It is clear that when $U_{0}$ is fixed and that the cathode voltage is decreased, the potential well becomes deeper and more efficient in trapping secondary electrons and hence the gun perveance is more strongly reduced (Fig. 9).

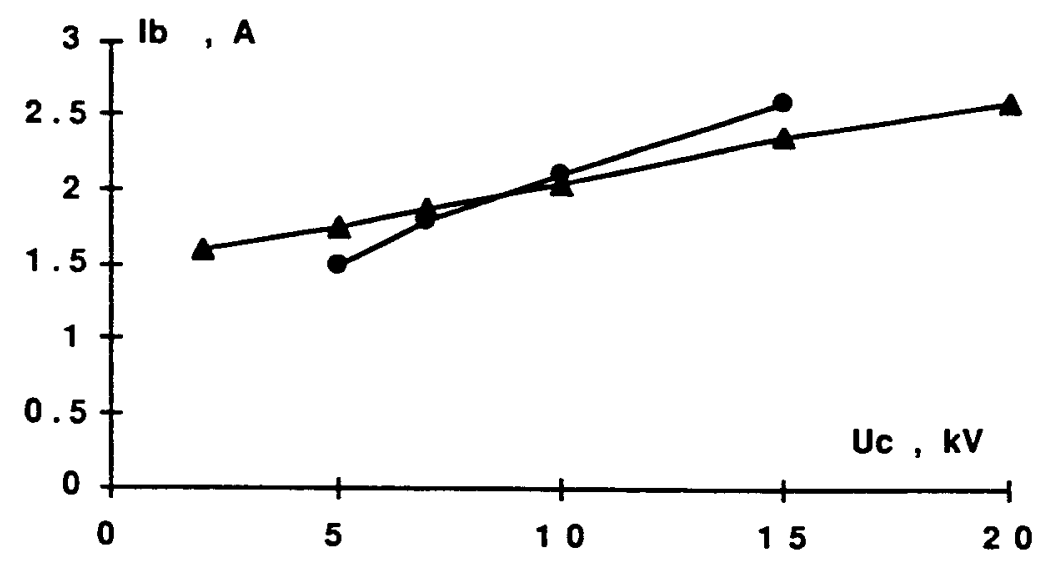

Fig. 9 - The dependence of beam current on the cathode potential when $U_{0}$ is fixed at $35 \mathrm{kV}$ $\left(I_{b}\right.$ should be equal to $2.7 \mathrm{~A}$ ). $\bullet:$ experimental curve, $\Delta:$ theoretical curve.

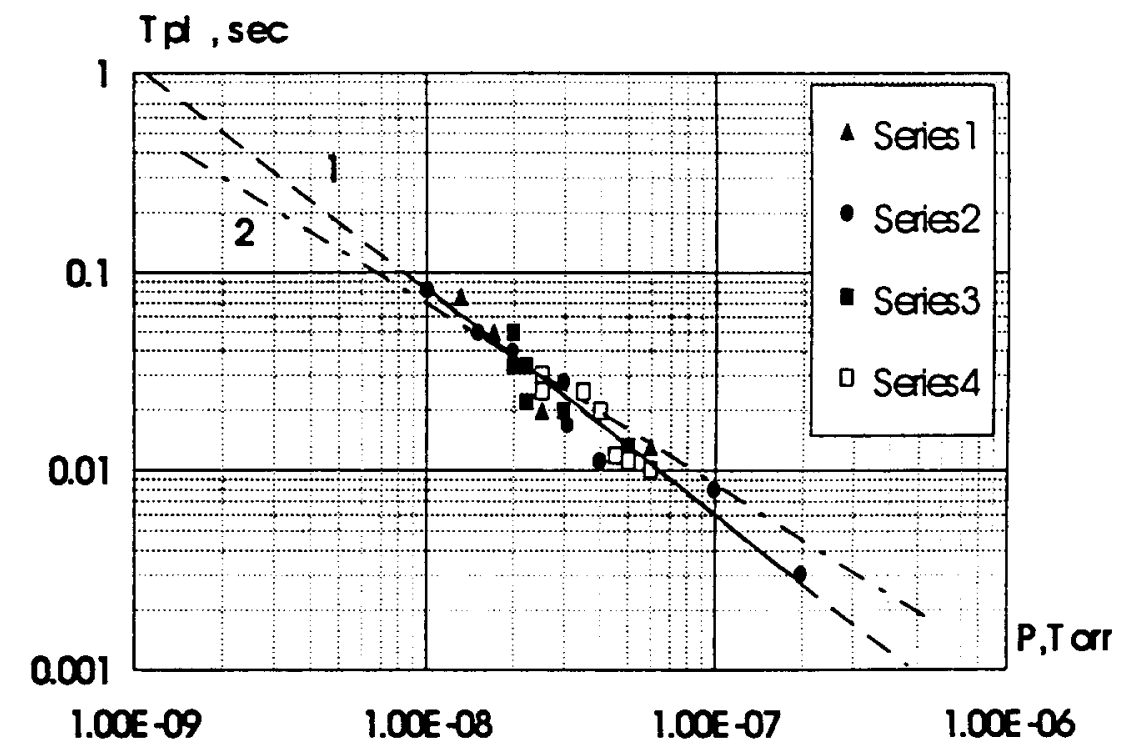

\begin{tabular}{|l|c|c|c|}
\hline & $U_{c}[\mathrm{kV}]$ & $U_{g}[\mathrm{kV}]$ & $I_{b}[\mathrm{~A}]$ \\
\hline Series 1 & 7 & 10 & 1.15 \\
\hline Series 2 & 7 & 15 & 1.6 \\
\hline Series 3 & 7 & 20 & 2.2 \\
\hline Series 4 & 15 & 15 & 2.5 \\
\hline
\end{tabular}

Fig. 10 - The dependence of the pulser period on the vacuum pressure. 1) experiment, 2) calculations $\left(U_{c}=7 \mathrm{kV}, U_{g}=10 \mathrm{kV}\right)$. 
It was also found that the gun perveance was not at all influenced by the vacuum conditions in the regime where the grid was at negative potential. However, in the regime where this potential is positive, it was shown that the vacuum pressure greatly affected the repetition period of the pulser in order to obtain a continous gun perveance greater than $90 \%$ of the design value. This threshold value for the pulser period was found to be inversely proportional to the vacuum pressure (Fig. 10 ):

$$
\begin{gathered}
T_{p l}(\mathrm{sec})=1 / 10^{9} P_{\text {Torr }} \\
8 \times 10^{-9} \text { Torr }<P<5 \times 10^{-6} \text { Torr }
\end{gathered}
$$

The secondary electron storage time $\tau_{s t}$ was also measured by observing the primary beam current variation after a single pulse. It was found that when the pulser period was made to be more or less equal to the storage time, $T_{p l} \approx \tau_{s t}$ the beam current only increased to $90 \%$ of the design value. This result indicates that there exists two groups of stored electrons, one with a short lifetime and one with a longer lifetime. The long lifetime group contains electrons oscillating between the collector and the cathode, while the short lifetime group those trapped in the potential well in the gun. The pulser cleans this well and the stored electrons then begin to oscillate in the electron cooling system.

\section{THE INFLUENCE OF STORED ELECTRONS ON THE GUN OPERATION}

We will now discuss the influence of the space charge of the stored electrons on the gun operation. If the grid is at a positive potential with respect to ground, the potential distribution will have a maximum value at a distance $d$ from the cathode (Fig. 11).

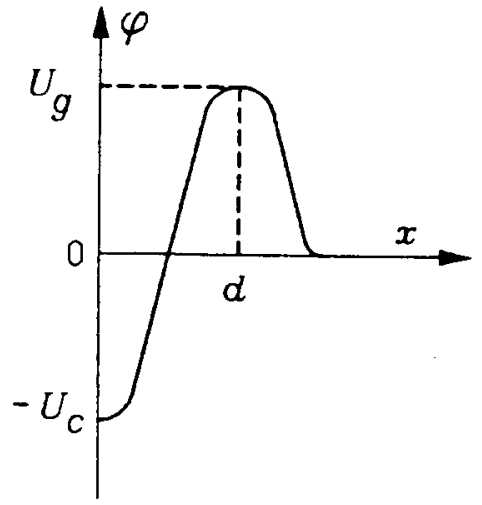

Fig. 11 - The potential distribution on the beam axis

The beam current is defined by the voltage difference between the cathode and the grid and also on the distance $d$ between these two, and is given by

$$
I_{b o}=\sqrt{\frac{2 e}{m}} \frac{a^{2}}{9 d^{2}} U_{0}^{3 / 2}
$$


where $U_{0}=U_{c}+U_{g}$ and $a$ is the beam radius $(a=25 \mathrm{~mm})$.

Secondary electrons are stored in this volume when the potential is positive and their density is given by the one dimensional Poisson equation

$$
\begin{aligned}
\frac{d^{2} \varphi}{d x^{2}} & =4 \pi e\left[n_{b}(\varphi)+n(\varphi)\right] \\
\left.\frac{d \varphi}{d x}\right|_{x=0} & =0,\left.\varphi\right|_{x=0}=-U_{c},\left.\varphi\right|_{x=d}=U_{g}
\end{aligned}
$$

where

$$
n_{b}(\varphi)=\frac{J_{b}}{e} \sqrt{\frac{m}{2 e\left(U_{c}+\varphi\right)}}, \quad J_{b}=\frac{I_{b}}{\pi a^{2}}
$$

is the electron beam density at a point where the potential is $\varphi(x)$ and $n(\varphi)$ the stored electron density

$$
n(\varphi)= \begin{cases}n(\varphi), & \varphi>0 \\ 0, & \varphi<0\end{cases}
$$

For convenience we use the dimensionless parameters for potential $\psi(x)$, coordinate $x_{0}$, and stored electron density $\xi(x)$ :

$$
\begin{aligned}
& \psi=\frac{\varphi(x)}{U_{0}}, U_{0}=U_{c}+U_{g}>0, \\
& x_{0}=\frac{x}{d}, \quad \xi\left(x_{0}\right)=\frac{n(x)}{n_{d}}
\end{aligned}
$$

Here $n_{d}$ is the beam density at $x=d$.

After integrating Eq. (5) with the given boundary conditions, one obtains the beam current delivered by the gun with stored electrons

$$
I_{b}=I_{b o}\left[\left(\frac{U_{c}}{U_{0}}\right)^{3 / 4}+\frac{3}{4} \int_{0}^{U_{g} / U_{0}} \frac{d \psi}{\left\{\left[\psi+\left(U_{c} / U_{0}\right)\right]^{1 / 2}+1 / 2 \int_{0}^{\psi} \xi\left(\psi^{\prime}\right) d \psi^{\prime}\right\}^{1 / 2}}\right]^{2}
$$

This formula gives two extreme values of the current

$$
I_{b}= \begin{cases}I_{b o}, & \xi<1, \\ I_{b o}\left(\frac{U_{c}}{U_{0}}\right)^{3 / 2}, & \xi>1\end{cases}
$$

(in the first case one can neglect the integral in the denominator of the second term in formula (8), and in the second case the second term itself). 
In the absence of stored electrons when $\xi \ll 1$, the beam current is defined by Eq. (4). If the stored electron density is sufficiently important, $\xi \gg 1$, then the beam current is limited by the space charge of the stored electrons and is given by

$$
I_{b}=I_{b o}\left(\frac{U_{c}}{U_{0}}\right)^{3 / 2}
$$

which only depends on the cathode potential.

The beam current limitation is charaterized by the following parameters of secondary electron beam dynamics:

1. the ionization time $\tau_{i}$,

2. the scattering time of an electron in a collision with residual gas atoms $\tau_{s c}$,

3. the electron escape time $\tau_{c s c}$, and

4. the pulser period $T_{p l}$.

The ionization time is defined as the time it takes for the secondary electron density to be equal to the density of the primary beam electrons

$$
\tau_{i}=\frac{1}{N \sigma v_{d}}
$$

Here $N$ is the residual gas density, $\sigma$ he ionization cross section of the primary beam electrons, and $v_{d}$ the electron beam velocity at the grid.

The scattering time is equal to

$$
\tau_{s c}=t_{o s c} \frac{1}{\left\langle\theta^{2}\right\rangle},\left\langle\theta^{2}\right\rangle=\frac{4 \pi e^{4} Z^{2} N d \Lambda_{1}}{m^{2} v^{4}}
$$

where $t_{\text {osc }}$ is the electron oscillation period in the gun, $\left\langle\theta^{2}\right\rangle$ the root mean square multiscattering angle of the stored electron, $e Z$ the nuclear charge of the residual gas atoms, $m$ the electron rest mass, $\Lambda_{1}$ the Coulomb logarithm, and $d$ the distance between the cathode and the grid (Fig. 11).

The escape time for stored electrons is defined by their diffusion in the magnetic field

$$
\tau_{e s c}=t_{o s c} \frac{a^{2}}{\rho_{h}^{2}\left(\theta^{2}\right)}
$$

In the formula $\rho_{h} \sqrt{\left\langle\theta^{2}\right\rangle}$ is the diffusion displacement of the stored electron in the magnetic field during one pass and $\rho_{h}$ the Larmor radius which is defined by the magnetic field $\left(\rho_{h}=\right.$ $\left.v_{d} / \omega_{h}, \omega_{h}=e B / m c\right)$. As in the present experimental conditions the electrons are magnetized,

$$
a \gg \rho_{h}
$$


the escape time is therefore much longer than the scattering time

$$
\tau_{e s c} \gg \tau_{s c}
$$

Two specific regimes of gun operation exist for different values of the pulser period $T_{p l}$. The first regime corresponds to a short pulser period value

$$
T_{p l} \ll \tau_{s c}<\tau_{e s c}
$$

In this case secondary scattering cannot occur between two successive pulses and the gun is continuously "cleaned" of stored electrons. The second regime occurs when the pulser period is long

$$
T_{p l} \gg \tau_{e s c} \gg \tau_{s c}
$$

In this situation scattering influences the velocity distribution of the stored electrons, becoming quasi uniform, and leads to the formation of a cloud of oscillating stored electrons. Both these regimes were experimentally observed and are discussed below.

\section{THE GUN OPERATION TOGETHER WITH THE PULSER}

We will now consider the operation of the gun when the pulser period is short so that Eq. (15) is respected. Again $\varphi>0$ and, therefore, $U_{g}>0$.

To the first order (see Appendix 1) the stored electron relative density versus time is given by:

$$
n(\psi, t)=n_{d} S(t) \sqrt{\psi}
$$

where

$S(t)=N \sigma_{0} v_{0} t\left(\frac{U_{0}}{U_{c}}\right)^{1 / 2}\left(\frac{U_{0}}{U_{g}}\right)^{1 / 2}$

$\sigma_{0}$ : ionization cross section at $x=d$,

$v_{0}: \sqrt{2 e U_{0} / m}$

Inserting (17) into (8) one then obtains the dependence of beam current on time

$$
I_{b}(t)=I_{b o}\left[\left(\frac{U_{c}}{U_{0}}\right)^{3 / 4}+\frac{3}{4} \int_{0}^{U_{g} / U_{0}} \frac{d \psi}{\left\{\left[\psi+\left(U_{c} / U_{0}\right)\right]^{1 / 2}+\left[(S(t) / 3)(\psi)^{3 / 2}\right]\right\}^{1 / 2}}\right]
$$

With this formula we are able to estimate the time it takes for the beam current to decrease to the threshold value of $0.95 I_{b o}$ defined earlier. This gives the threshold value for the pulser period $T_{p l}$. Figure 10 shows the dependence of the pulser period on the vacuum and one sees that the curves agree well with the measured pulser periods. 


\section{THE STATIONARY REGIME OF GUN OPERATION WITH STORED ELECTRONS}

In the stationary regime of gun operation, if the grid has a positive potential with respect to ground and the pulser is not used, the escape time of the stored electrons is longer than the scattering time (see Eq. (16)). The stored electron density in this case has been calculated in Appendix 2 and is equal to

$$
n(\psi)=n_{b} S_{1} \psi^{3 / 2}
$$

where

$$
S_{1}=\frac{\sigma_{0}}{3 \sigma_{e}}\left(\frac{\omega_{h} a}{c}\right)^{2} \frac{U_{0}}{U_{g}}\left(\frac{U_{0}}{U_{c}}\right)^{1 / 2}\left(\frac{\beta_{0}^{2}}{Z}\right)^{2} \frac{1}{\Lambda_{1} \Lambda_{2}},
$$

$\sigma_{e}=\pi\left(e^{2} / m c^{2}\right)^{2}$ is the cross section for electrons, $\beta_{0}=v_{0} / c$, and $\Lambda_{2}$ a logarithmic value, $\omega_{h}=$ $e B / m c$.

For the given function of $n(\psi)$ one can find the beam current

$$
I_{b}(t)=I_{b o}\left[\left(\frac{U_{c}}{U_{0}}\right)^{3 / 4}+\frac{3}{4} \int_{0}^{U_{8} / U_{0}} \frac{d \psi}{\left.\left\{\left[\psi+\left(U_{c} / U_{0}\right)\right]^{1 / 2}+\left[\left(S_{1}\right) / 5\right)(\psi)^{5 / 2}\right]\right\}^{1 / 2}}\right]
$$

The calculated dependence of the beam current on the cathode potential and the experimental values are in good agreement for $U_{0}=35 \mathrm{kV}$ and is shown in Fig. $9\left(Z=7, \Lambda_{1}=10\right.$, $\left.\sigma_{0}=2.5 \times 10^{-18} \mathrm{~cm}^{2}\right)$. It is the proof that the beam current limitation can be explained by the influence of stored electrons.

We see therefore that there are two distinct regions for the operation of the gun with full perveance. The boundary between these two regimes can be found from Eq. (20) by calculating the values of $U_{c}$ and $U_{g}$ for fixed values of $U_{0}$ with $I_{b}=0.95 I_{b o}$. The result of such a calculation is shown in Fig. 8. We see that to the right of the boundary the influence of the space charge of the stored electrons is important and that in order to reach full perveance the pulser has to be used.

\section{THE MEASUREMENT OF THE ELECTRON TRANSVERSE VELOCITY}

The electron transverse energy $E_{\perp}$ in the beam was measured using two different methods: an energetic [12] and an optical [13] analysis of small parts of the beam obtained by passing the beam through a cutting diaphragm. A detailed description of the method and the experimental setup can be found in Ref. [15]. The measured values of $E_{\perp}$ that we obtained did not exceed 0.3 to $0.7 \mathrm{eV}$ for beams with a perveance between 1.5 and $5 \mu \mathrm{A} / \mathrm{V}^{3 / 2}$ in a longitudinal magnetic field of $400 \mathrm{G}$. 


\section{TECHNICAL PROBLEMS RELATED TO THE GUN}

One severe problem, inherent to such a gun, is the problem of Penning discharge. Even after a bake-out of the whole system at $300^{\circ} \mathrm{C}$ at a vacuum pressure of $8 \times 10^{-8}$ Torr such a discharge existed due to the creation of Penning traps when the grid potential was positive with respect to ground.

To avoid this discharge a training of the gun was made. It consisted in slowly increasing the cathode and grid potentials and waiting for the discharge current to disappear before once again raising the potentials. Usually a training was necessary each time the gun was opened and exposed to atmospheric pressure. After a complete training cycle the gun was able to operate without any Penning discharge. It should be underlined that Penning discharge generation is very critical on the cleanliness of the electrode surfaces, such that once the gun had undergone the CERN vacuum cleaning procedures, Penning discharge was no longer observed.

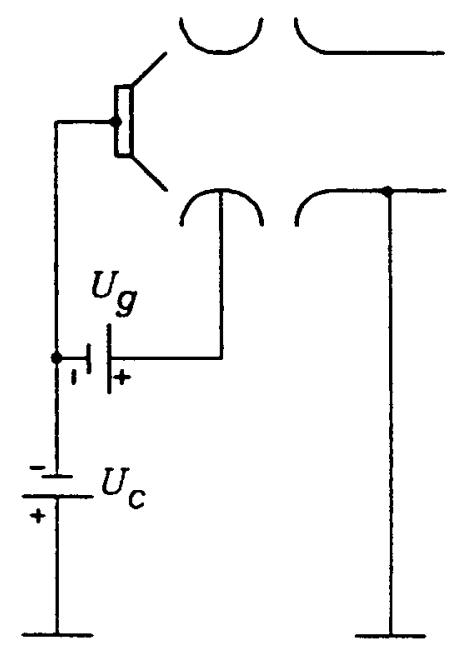

Fig. 12 - High voltage scheme for protection against high voltage breakdowns

The second problem is accidental high voltage breakdown. A breakdown of the cathode power supply is not dangerous as no electrons can be accelerated even if the grid potential is positive with respect to ground. However, if there is a breakdown in the grid power supply, then electrons can be lost anywhere in the system. These problems can be avoided if the high voltage scheme shown in Fig. 12 is used. As the grid potential is now with respect to the cathode, any power supply failure results in the immmediate decrease of the electron beam current and no losses can occur. This scheme is used on the LEAR electron cooler.

\section{FIRST EXPERIMENTS WITH THE ELECTRON GUN AT LEAR}

The new gun was mounted on the LEAR electron cooling device in early 1993 and the first gun tests and electron cooling experiments were performed immediately after. 
One of the goals of this experiment was to test the joint operation of the gun and the collector as the collector has a different design to the one used on the CAPT test bench and is not optimized for the recuperation of high intensity low energy beams. It was soon demonstrated that this was not a source of concern.

The LEAR electron cooler has two significant differences from the CAPT setup: a vacuum pressure of 10 to 50 pTorr and an S shaped solenoid with toroid magnets which produce an additional axial drift of the secondary electrons.

Table 3 shows that the gun perveance depends on the cathode and grid potentials as in the CAPT tests. The dependence of the gun perveance on the cathode potential is shown in Fig. 13. Here are represented the results of the calculations made using Eq. (20) for $Z=7, \Lambda_{1}=10$. The difference between theory and experiment for low cathode potentials is due to the influence of the tube perveance. The threshold value of the grid potential was found to be the same as in the CAPT tests (Fig. 10 ).

Table 3 - The measured electron gun characteristics for the LEAR tests

\begin{tabular}{|c|c|c|c|c|}
\hline Electron energy & $(\mathrm{keV})$ & 7 & 7 & 7 \\
\hline Beam current & (A) & 2.8 & 1.7 & 1.4 \\
\hline Beam perveance & $\left(\mu \mathrm{A} / \mathrm{V}^{3 / 2}\right)$ & 4.8 & 2.7 & 2.3 \\
\hline Gun perveance & $\left(\mu \mathrm{A} / \mathrm{V}^{3 / 2}\right)$ & 0.55 & 0.56 & 0.49 \\
\hline $\begin{array}{l}\text { Voltage difference betwee } \\
\text { grid and cathode: } U_{0}\end{array}$ & (kV) & 30 & 20 & 20 \\
\hline Current losses & $(\mathrm{mA})$ & 1.8 & 0.2 & 0.25 \\
\hline Collector potential & $(\mathrm{kV})$ & 3.05 & 2.9 & 3 \\
\hline Repeller potential & $(\mathrm{kV})$ & 2.3 & 1.5 & 1 \\
\hline Magnetic field & (G) & 600 & 600 & 600 \\
\hline Pulser repetition period & (s) & 600 & 3 & 6 \\
\hline $\begin{array}{l}\text { Vacuum pressure } \\
\text { in the gun } \\
\text { in the collector }\end{array}$ & & $\begin{array}{l}30 \\
60\end{array}$ & $\begin{array}{l}20 \\
30\end{array}$ & $\begin{array}{l}12 \\
15\end{array}$ \\
\hline
\end{tabular}

Some differences in the gun operation at LEAR were nevertheless observed. The collector tuning did influence the gun perveance and when kept to an optimum the beam perveance could be kept at $P_{b}=5 \mu \mathrm{A} / \mathrm{V}^{3 / 2}$ for periods of 20 minutes or more.To operate in such a regime the grid potential was increased to the desired value and a single pulse was delivered to the grid through the pulser. In this way the full perveance was established during a 10 to 20 minutes period after which a sudden jump in current lowered the gun perveance to its previous value. By applying another pulse full perveance was once again reached. 


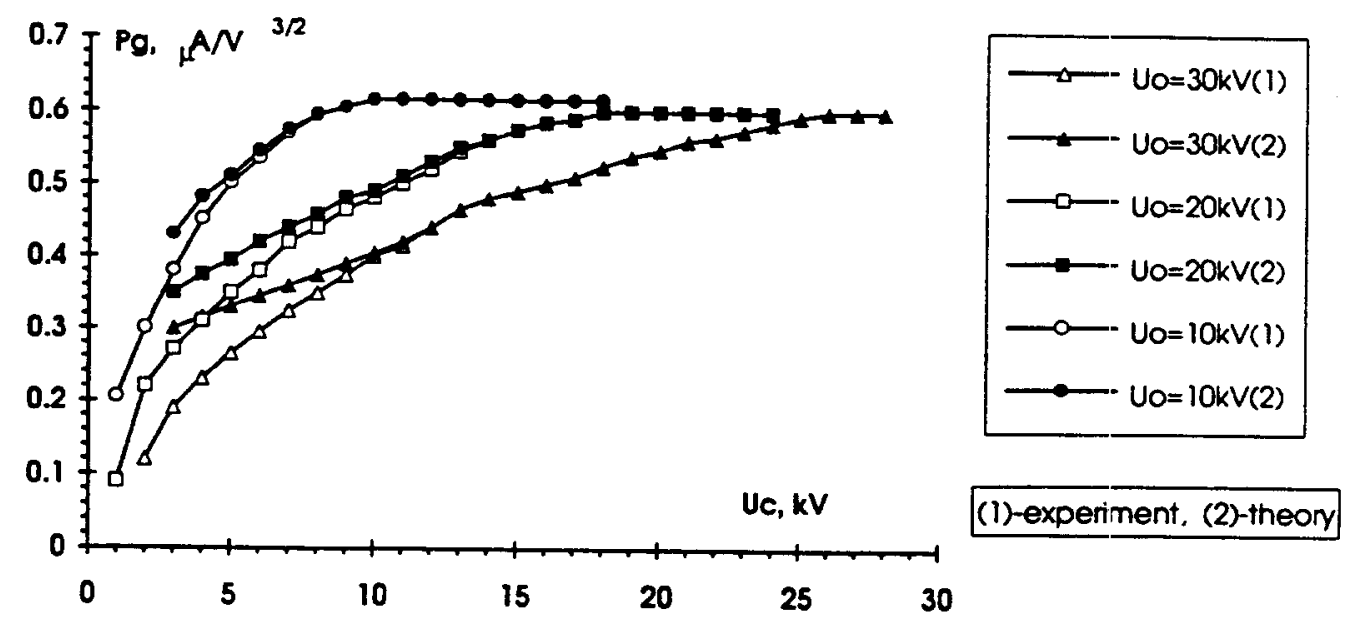

Fig. 13 - Measured gun perveance as a function of $U_{c}$

The results of the first electron cooling experiments with the new gun are summarized in Table 4 but presented elsewhere [16]. It should be mentioned that these experiments were performed with the standard regimes of beam perveance equal to $P_{b}={ }^{0} 5 \mu \mathrm{A} / \mathrm{V}^{3 / 2}$. The use of higher beam perveances were not fully investigated except in the pulsed mode regime. Nonetheless, some advantages of the new gun were felt, especially the possibility to independently regulate the electron beam current.

\section{CONCLUSIONS}

The variable current gun for the LEAR electron cooler has been designed, constructed and tested up to electron energies of $35 \mathrm{keV}$ and beam currents of $3.3 \mathrm{~A}$. A beam perveance of $5 \mu \mathrm{A} / \mathrm{V}^{3 / 2}$ has been obtained using an adiabatic optics scheme in a relatively low magnetic field.

Table 4 - Main parameters for the electron cooling experiments

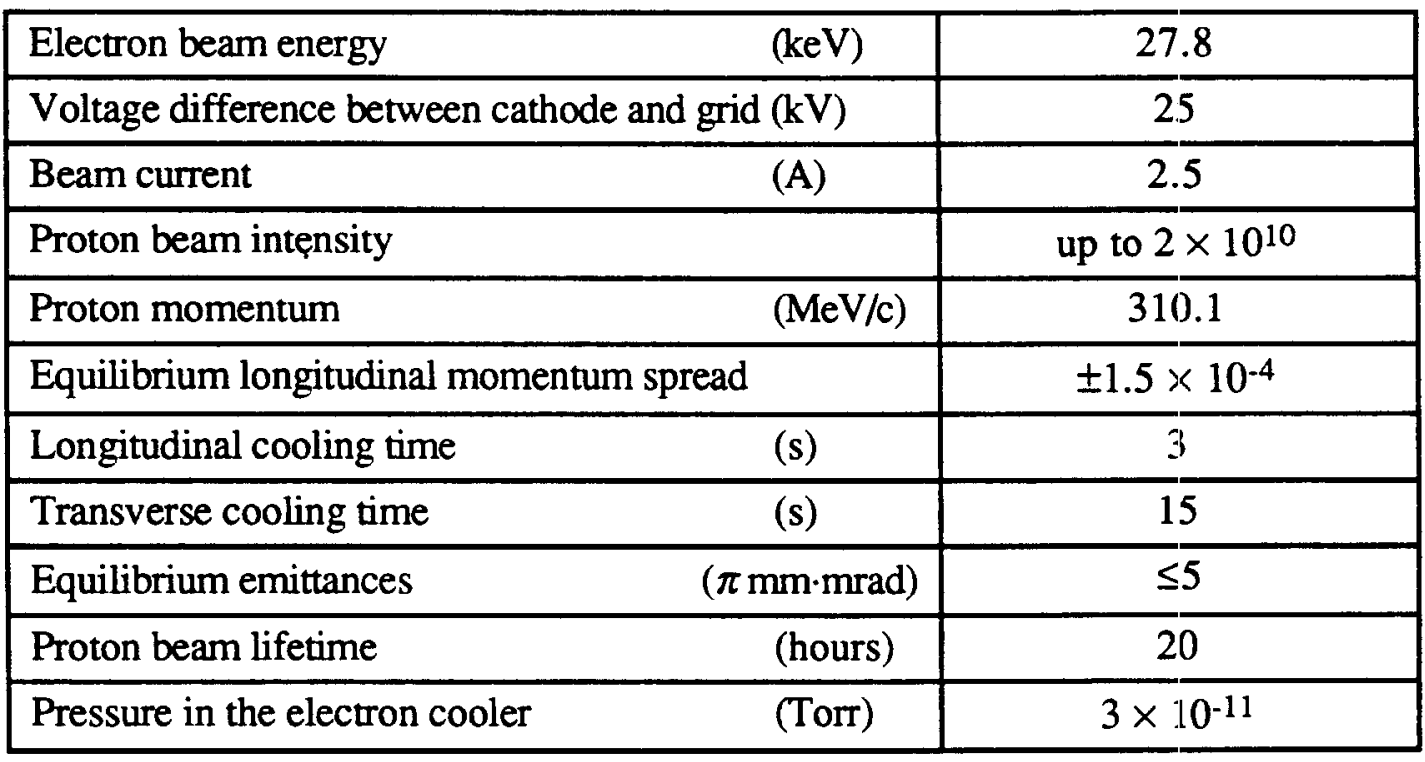




\section{ACKNOWLEDGEMENTS}

The authors are grateful to A. Skrinsky, K. Hübner, S. Maury, D. Möhl, G. Molinari,

L. Montanet, and C. Vasseur for their effective support of this work.

\section{REFERENCES}

[1] G.I. Budker, A.N. Skrinsky, Electron Cooling and New Perspective in the Physics of Elementary Particles. UFN, 1979, N 124, p. 561.

[2] Y. Derbehev, I. Meshkov, Studies on Electron Cooling of Heavy Particle Beams Made by the VAPP-NAP Group at the Nuclear Physics Institute of the Siberian Branch of the USSR Academy of Science at Novosibirsk, CERN 77-08 (1977).

[3] H. Poth, Electron Cooling, Theory Experiment and Application, CERN EP 90-04 (1990).

[4] V.A. Lebedev, A.N. Sharapa, Formation of an Electron Beam With Small Transverse Velocity in a System With a Longitudinal Magnetic Field, JTF.V57.N5, p. 975.

[5] V.I. Kudelainen, I.N. Meshkov, R.A. Salimov, Formation of an Intense Electron Beam in a Longitudinal Magnetic Field, JTF.V41.N11, p. 2294.

[6] J. Bosser, I. Meshkov, V. Poliakov, A. Smirnov, E. Syresin, G. Tranquille, A. Zapunjako, Project for a Variable Current Electron Gun for the LEAR Electron Cooler, CERN PS/92-03 (AR), (1992).

[7] J. Bosser, R. Ley, G. Tranquille, V. Bykovsky, V. Funtikov, I. Meshkov, A. Rogozin, V. Poliakov, I. Seleznev, V. Sinitsky, A. Smirnov, E. Syresin, The New Collector for the Electron Cooling Device at LEAR, NIM A311 (1992), p. 465.

[8] I. Meshkov, S. Agishev, V. Bykovsky, V. Poliakov, I. Seleznev, A. Smirnov, E. Syresin, The Electron Cooling System of Complex K4-K10, Published in 'Heavy ion storage complex K4-K10' (1992).

[9] V.I. Kudelainen, I.N. Meshkov, V.V. Parkhomchuk, R. Salimov, A. Skrinsky, V. Fainstein, JETP, 1976, N46, p. 1678.

[10] A.V. Burov, V. Kudelainen, V. Lebedev, V.V. Parkhomchuk, A. Sery, V. Shiltsev, Experimental Investigation of an Electron Beam in a Compensated State, Preprint INP 89-116, 1989 and CERN PS 93-03(AR) (1993).

[11] J. Bosser, D. Möhl, G. Tranquille, I. Meshkov, E. Syresin, V.V. Parkhomchuk, Neutralization of the LEAR Ecool Electron Beam Space Charge, CERN PS/93-41 (AR) (1993).

[12] N. Dikansky, V. Kudelainen, V. Lebedev, I. Meshkov, V.V. Parkhomchuk, Ultimate Possibilities of Electron Cooling, INP 88-61, 1988.

[13] V. Ginkin, I. Meshkov, A. Skrinsky, V. Fainstein, PTE, N6, p. 26, 1972.

[14] G. Stupakov, E. Syresin, Formation of Oscillating Electrons in Diode With Thin Anode Foil, Phisuca plasma, 1986, N1, p. 81.

[15] J. Bosser, R. Ley, G. Tranquille, V. Golubev, R. Lapik, I. Meshkov, V. Mozgunov, V. Poliakov, I. Seleznev, A. Smirnov, E. Syresin, M. Zavrazhnov, The Measurement of Transversal and Longitudinal Velocities of an Electron Beam, Proceedings of the 'Workshop on Beam Cooling and Related Topics', CERN Report 94-03, 1994.

[16] J. Bosser, R. Ley, G. Tranquille, R. Lapik, I. Meshkov, V. Poliakov, I. Seleznev, A. Smirnov, E. Syresin, A. Zapunjako, The First Results of Electron Cooling at LEAR With the Variable Current Electron Gun, Proceedings of the 'Workshop on Beam Cooling and Related Topics', CERN Report 94-03, 1994. 


\section{Appendix 1}

For simplicity, we consider that around $x=d$ the potential distribution $\varphi$ can be approximated by the Child-Langmuir law (dotted line in Fig. A.1),

$$
\varphi=U_{g}-U_{0}\left[\frac{|x-d|}{d}\right]^{4 / 3}
$$

such as $\phi=0$ for

$$
x=x_{a, b}=d\left[1 \pm\left(\frac{U_{g}}{U_{0}}\right)^{3 / 4}\right]
$$

We can therefore make use of our normalization factor

$$
\psi=\frac{\varphi}{U_{0}}=\frac{U_{g}}{U_{0}}-\left[\frac{|x-d|}{d}\right]^{4 / 3}
$$

and set $\varphi_{1}=\varphi\left(x=x_{1}\right)$ and $\varphi_{d}=\varphi(x=d)=U_{g}$.

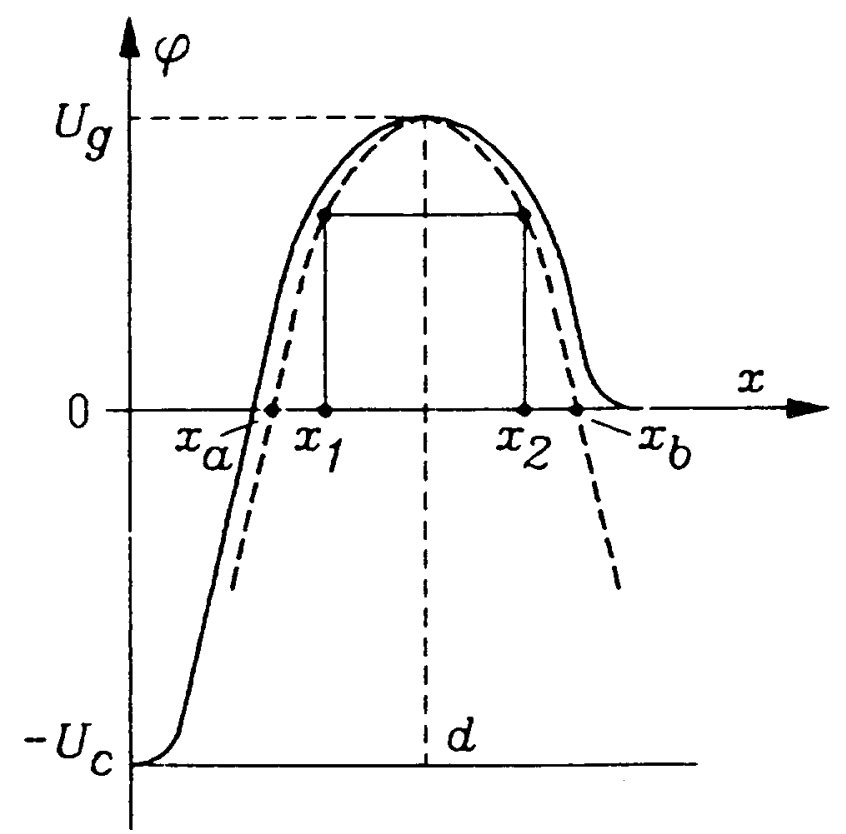

Fig. A.1 - Potential distribution at the neighbourhood of the grid

\section{Oscillation Period}

An electron ionized at $x_{1}$ (and therefore at rest at $x_{1}$ ) will oscillate between $x_{1}$ and its symmetric point $x_{2}$, with respect to $d$, with an oscillation period $t_{\text {osc }}$ that we will estimate. At any point $\mathrm{x} \in\left[x_{1}, x_{2}\right]$ the ionized electron velocity is given by 


$$
\frac{d x}{d t}=\sqrt{\frac{2 e}{m}} \cdot \sqrt{\varphi(x)-\varphi\left(x_{1}\right)}
$$

Then

$$
t_{o s c}=\frac{4}{\sqrt{2 e / m}} \int_{x_{1}}^{d} \frac{d x}{\sqrt{\varphi(x)-\varphi\left(x_{1}\right)}}
$$

In the range of integration

$$
\begin{gathered}
d x=\frac{3 d}{4 U_{0}}\left[\frac{\varphi-U_{g}}{U_{0}}\right]^{-1 / 4} d \varphi . \\
t_{o s c}=\left.\frac{3 d}{\sqrt{2 e U_{0} / m} \cdot \sqrt{U_{0}}} \int_{\varphi_{1}}^{\varphi_{d}} \frac{d \varphi}{\left.\left[\varphi-U_{g}\right) / U_{0}\right]^{1 / 4} \sqrt{\varphi-\varphi_{1}}} \cong \frac{6 d}{\sqrt{2 e U_{0} / m}}\left[\frac{\varphi-\varphi_{1}}{U_{0}}\right]^{1 / 4}\right|_{\varphi_{1}} ^{\varphi_{d}} \\
t_{\text {osc }} \cong \frac{6 d}{v_{0}}\left[\psi_{d}-\psi_{1}\right]^{1 / 4} \text { with } v_{0}=\sqrt{\frac{2 e U_{0}}{m}} .
\end{gathered}
$$

\section{Stored Electron Density}

The electrons resulting from the ionization by the main current $I_{b}$ of the residual gas are initially at rest at $x_{1}$ or $x_{2}$. Owing to the potential well they will oscillate between $x_{1}$ and $x_{2}$. Their density is smallest at $x=d$ where their velocity $v_{d}=\sqrt{(2 e / m)\left(\varphi_{d}-\varphi_{1}\right)}$ is at its maximum.

The stored electron density at any point $x \in\left[x_{1}, x_{2}\right]$ at a time $t$, due to ionization of the residual gas atoms at a point $x_{1}$ is equal to:

$$
d n\left(x, x_{1}\right)=\frac{J_{b}}{e} \frac{N \sigma\left(x_{1}\right) d x_{1}}{v\left(x, x_{1}\right)} \frac{t}{\left(t_{\text {osc }} / 2\right)}
$$

with

$$
v\left(x, x_{1}\right)=\sqrt{\frac{2 e}{m}\left(\varphi(x)-\varphi_{1}\right)}=\sqrt{\frac{2 e U_{0}}{m}} \cdot \sqrt{\frac{\varphi(x)}{U_{0}}-\frac{\varphi_{1}}{U_{0}}}
$$

$J_{b}$ is the electron current density.

The electron beam energy depends on the coordinate $x$ of the electron inside the gun. On the other hand, the ionization cross section is inversely proportional to the ion velocity. Therefore, the dependence on $x_{1}$ can be taken as follows:

$$
\sigma\left(x_{1}\right)=\frac{\sigma_{0}}{\sqrt{\left(U_{c}+\varphi_{1}\right) / U_{0}}}
$$


where $\sigma_{0}$ is the ionization cross section at $\varphi_{d}$.

Using the expression of $t_{o s c}, v\left(x, x_{1}\right), \sigma\left(x_{1}\right)$ and integrating $d n\left(x, x_{1}\right)$ over the interval $\left[x, x_{1}\right]$

$$
n(x, t)=\left[\frac{J_{b} N \sigma_{0} t}{e}\right] \int_{x_{d}}^{x} \frac{d x_{1}}{\sqrt{\left(U_{c}+\varphi_{1}\right) / U_{0}}\left(3 d / v_{o}\right)\left(\left(\varphi_{d}-\varphi_{1}\right) / U_{0}\right)^{1 / 4} v_{0} \sqrt{\left(\varphi(x)-\varphi_{1}\right) / U_{0}}}
$$

but

$$
\begin{gathered}
d x_{1}=\frac{3 d}{4 U_{0}}\left[\frac{\varphi_{1}-\varphi_{d}}{U_{0}}\right]^{-1 / 4} d \varphi_{1} \\
n(\varphi, t)=\frac{J_{b} N \sigma_{0} t}{3 d e} \frac{2 \cdot 3 d}{4 U_{0}} \int_{0}^{\varphi} \frac{d \varphi_{1}}{\sqrt{\left(U_{c}+\varphi_{1}\right) / U_{0}} \sqrt{\left(\varphi_{d}-\varphi_{1}(x)\right) / U_{0}} \sqrt{\left(\varphi(x)-\varphi_{1}\right) / U_{0}}}
\end{gathered}
$$

Setting

$$
\begin{gathered}
\psi=\frac{\varphi}{U_{0}} \text { and } d \psi=\frac{d \varphi}{U_{0}} \\
n(\psi, t)=\frac{J_{b} N \sigma_{0} t}{2 e} \int_{0}^{\psi} \frac{d \psi_{1}}{\left[\left(U_{g} / U_{0}\right)-\psi_{1}\right]^{1 / 2}\left[\left(U_{c} / U_{0}\right)+\psi_{1}\right]^{1 / 2}\left(\psi-\psi_{1}\right)^{1 / 2}}
\end{gathered}
$$

For simplicity, we consider that:

$$
\left(\frac{U_{d}}{U_{0}}-\psi_{1}\right)^{1 / 2}\left(\frac{U_{c}}{U_{0}}+\psi_{1}\right)^{1 / 2} \cong\left(\frac{U_{g}}{U_{0}}\right)^{1 / 2}\left(\frac{U_{c}}{U_{0}}\right)^{1 / 2}
$$

then

$$
\begin{gathered}
n(\psi, t)=\frac{J_{b} N \sigma_{0} t}{2 e}\left(\frac{U_{0}}{U_{g}}\right)^{1 / 2}\left(\frac{U_{0}}{U_{c}}\right)^{1 / 2} \int_{0}^{\psi} \frac{d \psi_{1}}{\sqrt{\psi-\psi_{1}}} \\
n(\psi, t)=n_{0} S(t) \sqrt{\psi}
\end{gathered}
$$

where, since $J_{b}=n_{0} v_{0} e$

$$
S(t)=N \sigma_{0} v_{0} t\left(\frac{U_{0}}{U_{g}}\right)^{1 / 2}\left(\frac{U_{0}}{U_{c}}\right)^{1 / 2}
$$




\section{Appendix 2}

The density of stored electrons at a point with a potential $\psi$ can be calculated for a given function of momentum distribution [14]. The momentum distribution function is determined by the ionization of gas atoms by the electron beam, scattering of secondary electrons on gas atoms, and their escape across to the magnetic field.

Let us calculate the distribution function $f(P, \theta)$ at the point $x=d$ for electrons which are generated at a point $x_{1}$. The kinetic equation for $f(P, \theta)$ can be written as follows:

$$
\frac{\partial f}{\partial t}=\frac{\xi_{0}}{\sin \theta} \frac{\partial}{\partial \theta} \sin \theta \frac{\partial f}{\partial \theta}+\frac{1}{r} \frac{\partial}{\partial r} r D \frac{\partial f}{\partial r}+\frac{d n_{i}}{4 \pi P^{2} t_{\text {osc }}} \delta\left(P-P_{d}\right) \frac{\delta(\theta)}{\sin \theta}
$$

where $\theta$ is the angle between the directions of the electron momentum and the axis of the system, $\xi_{0}=\left\langle\theta^{2}\right\rangle / t_{\text {osc }}$ the frequency of secondary electron scattering on residual gas atoms, $t_{o s c}\left(x_{1}\right)$ one half-period of oscillation for the ionization electrons generated at the point $x_{1},\langle\theta\rangle 2$ the root-mean-square value of the multiscattering angle received by an electron for time duration $t_{\text {osc }}\left(x_{1}\right), r$ the radial coordinate of a secondary electron in the reference frame, whose axis coincides with the beam axis, $D=\xi_{0} \rho_{h}{ }^{2}$ the diffusion coefficient for secondary electrons, $d n_{i}=J_{b} N \sigma\left(x_{1}\right) d x_{1} / e v_{d}\left(x_{1}, d\right)$ the electron density at the point $x=d$ for an electron produced at a point $x_{1},\left(d x_{1}=(3 d / 4)\left(\left|\psi_{1}-\psi_{\mathrm{d}}\right|\right)^{-1 / 4} d \psi_{1}\right.$ is the same expression as Eq. (1.3) and must not be confused with the product $\left.d \cdot x_{1}\right), P_{d}=P_{0}\left[\left(U_{g} / U_{0}\right)-\psi_{1}\right]^{1 / 2}$ the momentum at the point $x=d$ for an electron produced at a point $x_{1}$ with a potential $\psi_{1}, v_{d}=P_{d} / \mathrm{m}, P_{0}=\sqrt{ } 2 e m U_{0}$.

One can solve the kinetic equation (2.1) for two limiting cases (15) and (16). When $T_{p l}<<\tau_{s c} \ll<\tau_{e s c}$ (condition (15)), one can neglect the first and the second terms on the righthand side of Eq. (2.1). The scattering and escape of secondary electrons in this case is very low. The momentum distribution is one dimensional $(\theta \equiv 0)$, and the kinetic equation (2.1) is described by the formula(the normalisation factor $4 \pi P^{2}$ is reduced to 1 )

$$
\frac{\partial f}{\partial t}=\frac{J_{b} N \sigma\left(x_{1}\right) d x_{1}}{e v_{d}\left(x_{1}, d\right) t_{o s c}\left(x_{1}\right)} \delta\left(P-P_{d}\right)
$$

From Eq. (2.2):

$$
f\left(P, x_{1}\right)=\frac{J_{b} N \sigma\left(x_{1}\right) t d x_{1}}{e t_{o s c}\left(x_{1}\right) v_{d}} \delta\left(P-P_{d}\right)
$$

As secondary electrons are stored, the variation of the potential distribution inside the gun is low during one period of the electron oscillation, because the distribution function depends only on the electron energy and on the coordinates of an electron generation point and does not depend on the $x$-coordinate. The stored density at a point $x$ [14] is equal to 


$$
d n\left(x, x_{1}\right)=\int f\left(P_{2}, x_{1}\right) d P_{2}
$$

where $P_{2}$ is the momentum at a point $x$ for an electron which has a momentum $P$ at the point $x=d$. It follows the equation of energy conservation

$$
\frac{P_{2}^{2}}{2 m}-e \varphi(x)=\frac{P^{2}}{2 m}-e U_{g}
$$

After substitution of formulae (2.3) and (2.5) in Eq. (4.2), one obtains

$$
d n\left(x, x_{1}\right)=\frac{J_{b} N \sigma\left(x_{1}\right) d x_{1}}{e v_{d}} \frac{t}{t_{o s c}\left(x_{1}\right)} \int \frac{\delta\left(P-P_{d}\right) P d P}{\sqrt{P^{2}-P_{0}^{2}\left[\left(U_{g} / U_{0}\right)-\psi\right]}} .
$$

The integration gives the electron density at a point $x$ with a potential $\varphi$

$$
d n\left(x, x_{1}\right)=\frac{J_{b} N \sigma\left(x_{1}\right) d x_{1} t}{e t_{o s c}\left(x_{1}\right) v\left(x, x_{1}\right)}
$$

where $v\left(x, x_{1}\right)=\sqrt{(2 e / m)\left[\varphi(x)-\varphi\left(x_{1}\right)\right]}$ is the velocity at a point $x$ of an electron generated at a point $x_{1}$. As a result, one comes to formula (17) since (2.7) is equivalent to (1.2) (Appendix 1).

Let us determine now the density of stored electrons for a stationary case, when condition (16), $T_{p l} \gg \tau_{e s c} \gg \tau_{s c}$, is respected. In this case, the left-hand side of Eq. (2.1) and the first part of the right-hand side in Eq. (2.1) are equal to zero. Let us multiply Eq. (2.1) by $\sin \theta$ and integrate over $\theta$ angles. After such an operation, one has

$$
\frac{1}{r} \frac{\partial}{\partial r} r D \frac{\partial f}{\partial r}+\frac{d n_{i}}{4 \pi P^{2} t_{o s c}} \delta\left(P-P_{d}\right)=0 .
$$

One can estimate by an order of magnitude

$$
\frac{1}{r} \frac{\partial}{\partial r} r D \frac{\partial f}{\partial r} \cong \frac{D f}{a^{2}}
$$

then the distribution function at the point $x=d$ for electrons generated at a point $x_{1}$ is as follows

$$
f=-\frac{a^{2}}{D\left(x_{1}\right)} \frac{d n_{i} \delta\left(P-P_{d}\right)}{t_{o s c}\left(x_{1}\right) 4 \pi P^{2}}=-\frac{a^{2}}{D\left(x_{1}\right)} \frac{J_{b} N \sigma\left(x_{1}\right) d x_{1}}{e v_{d} t_{o s c}\left(x_{1}\right)} \frac{\delta\left(P-P_{d}\right)}{4 \pi P^{2}} .
$$

The electron density at a point $x$ for an isotropic distribution [14] is equal to

$$
d n\left(x, x_{1}\right)=4 \pi \int f\left(P_{2}, x_{1}\right) P_{2}^{2} d P_{2}
$$

where $P_{2}$ is the electron momentum at a point $x$ determined by Eq. (2.5). After the transformation to the new parameters $P$, one can describe the density $d n\left(x, x_{1}\right)$

$$
d n\left(x, x_{1}\right)=4 \pi \int f\left(P, x_{1}\right) \sqrt{P^{2}-P_{0}^{2}\left[\left(U_{g} / U_{0}\right)-\psi(x)\right]} P d P
$$


and, after substitution of Eq. (2.9) in here, one obtains

$$
d n\left(x, x_{1}\right)=4 \pi \frac{J_{b} N \sigma\left(x_{1}\right) d x_{1} a^{2}}{e D\left(x_{1}\right) v_{d} t_{o s c}\left(x_{1}\right)} \int \frac{\delta\left(P-P_{d}\right)}{4 \pi P^{2}} \sqrt{P^{2}-P_{0}^{2}\left[\left(U_{g} / U_{0}\right)-\psi(x)\right]} P d P
$$

The diffusion coefficient is equal to

$$
D=\frac{2}{t_{o s c}\left(x_{1}\right)} \int_{x_{1}}^{d} \rho_{h}^{2}\left(x_{2}\right) \frac{4 \pi e^{4} N Z^{2} \Lambda_{1}}{\left(m^{2} v_{1}^{2}\left(x_{2}\right)\right)^{2}} d x_{2}
$$

where $\rho_{h}\left(x_{2}\right)=v_{1} / \omega_{h}, v_{1}=v_{0} \sqrt{ } \psi\left(x_{2}\right)-\psi\left(x_{1}\right)$ the velocity at a point $x_{2}$ for an electron generated at a point $x_{1}$. It follows the dependence $\psi(x)$ given by formula (1.1)

$$
D\left(x_{1}\right)=\frac{\rho_{0}^{2}}{t_{o s c}\left(x_{1}\right)} \frac{\theta_{0}^{2} \Lambda_{2}}{\left[\left(U_{g} / U_{0}\right)-\psi_{1}\left(x_{1}\right)\right]^{1 / 4}}
$$

where $\rho_{0}=v_{0} / \omega_{h}, \theta_{0}^{2}=3 \pi e^{4} Z^{2} N d \Lambda_{1} / m^{2} v_{0}^{4}, \Lambda_{2}=8(7 / 6-\ln 2)$.

To calculate the density of stored electrons at a point $x$ with a potential $\psi(x)$, Eq. (2.12) will be integrated over all $x_{1}$ points where secondary electrons are being generated. Using (1.3):

$$
n(\psi)=\frac{3}{2} \frac{n_{b} N \sigma_{0} d a^{2}}{\rho_{0}^{2} \theta_{1}^{2}} \int_{0}^{\psi} \frac{\sqrt{\psi-\psi_{1}} d \psi_{1}}{\left[\left(U_{g} / U_{0}\right)-\psi_{1}\right] \sqrt{\left[\left(U_{c} / U_{0}\right)+1\right]}}
$$

where $\theta_{1}^{2}=\theta_{0}^{2} \Lambda_{2} \cdot n(\psi)$ can be represented in an analogue manner as (1.4). After integration of Eq. (2.15), one gets the density of secondary electrons with an accuracy up to $\psi^{5 / 2}$.

$$
n(\psi)=n_{b} S_{1} \psi^{3 / 2}
$$

where

$$
S_{1}=N \sigma_{0} d \frac{U_{0}}{U_{g}}\left(\frac{U_{0}}{U_{c}}\right)^{1 / 2} \frac{a^{2}}{\rho_{0}^{2} \theta_{1}^{2}}=\frac{\sigma_{0}}{3 \sigma_{e}} \frac{\omega_{h}^{2} a^{2}}{c^{2}} \frac{U_{0}}{U_{g}}\left(\frac{U_{0}}{U_{c}}\right)^{1 / 2} \frac{\beta_{0}^{2}}{Z^{2} \Lambda_{2} \Lambda_{1}}
$$

$\sigma_{e}=\pi\left(e^{2} / m c^{2}\right)^{2}$ the cross section for electrons, $\beta_{0}=v_{0} / c$. 
